PROCEEDINGS OF THE

AMERICAN MATHEMATICAL SOCIETY

Volume 127, Number 1, January 1999, Pages 223-233

S 0002-9939(99)04555-4

\title{
THE S-ELEMENTARY WAVELETS ARE PATH-CONNECTED
}

\author{
D. M. SPEEGLE
}

(Communicated by J. Marshall Ash)

\begin{abstract}
A construction of wavelet sets containing certain subsets of $\mathbb{R}$ is given. The construction is then modified to yield a continuous dependence on the underlying subset, which is used to prove the path-connectedness of the s-elementary wavelets. A generalization to $\mathbb{R}^{n}$ is also considered.
\end{abstract}

\section{INTRODUCTION}

A function $f \in L^{2}(\mathbb{R})$ is a dyadic orthogonal wavelet (or simply a wavelet if no confusion can arise) if $\left\{2^{n / 2} f\left(2^{n} x+l\right)\right\}_{l, n \in \mathbb{Z}}$ is an orthonormal basis for $L^{2}(\mathbb{R})$. Alternatively, if we define $D: L^{2}(\mathbb{R}) \rightarrow L^{2}(\mathbb{R})$ by $D(f)(t)=\sqrt{2} f(2 t)$ and $T$ : $L^{2}(\mathbb{R}) \rightarrow L^{2}(\mathbb{R})$ by $T(f)(t)=f(t-1)$, then by definition $f$ is a wavelet if and only if $f$ is a complete wandering vector for the unitary system $\mathcal{U}=\left\{D^{n} T^{l}\right\}_{l, n \in \mathbb{Z}}$, written $f \in \mathcal{W}(\mathcal{U})$. In operator theory, the set $\mathcal{W}(\mathcal{V})$ has been studied mostly in the case that $\mathcal{V}$ is a singly generated infinite group of unitaries $[\mathrm{H}]$. In our case, however, $\mathcal{U}$ is not even a semigroup, and much less is known about the structure of $\mathcal{W}(\mathcal{U})$. For example, while it is known that $\mathcal{W}(\mathcal{U})$ is not closed but still has dense span, it is not known whether $\mathcal{W}(\mathcal{U})$ is connected, i.e. pathwise in the $L^{2}(\mathbb{R})$ metric. However, Dai and Larson $[\mathrm{DL}]$ showed that $\mathcal{W}(\mathcal{U})$ does not have any trivial components.

Now, if we define $\hat{D}=\mathcal{F} D \mathcal{F}^{-1}=D^{-1}$ and $\hat{T}=\mathcal{F} T \mathcal{F}^{-1}=M_{e^{-i s}}$, where $\mathcal{F}$ is the Fourier transform and $M_{g}$ is multiplication by $g$, then $f$ is a wavelet if and only if $\hat{f} \in \mathcal{W}(\hat{\mathcal{U}})=\mathcal{W}\left(\left\{\hat{D}^{n} \hat{T}^{l}\right\}_{l, n \in Z}\right)$. One example of such a function is $\frac{1}{\sqrt{2 \pi}} 1_{E}$, where $E=[-2 \pi,-\pi) \cup[\pi, 2 \pi)$. It is not hard to see that when $W$ is measurable, $\frac{1}{\sqrt{2 \pi}} 1_{W}$ is the Fourier transform of a wavelet if and only if (modulo null sets)

$$
\bigcup_{i=-\infty}^{\infty} 2^{i} W=\mathbb{R}, \quad \bigcup_{i=-\infty}^{\infty}(W+2 \pi i)=\mathbb{R}
$$

with both unions disjoint. Following Dai and Larson, we call such a set $W$ a wavelet set and the inverse Fourier transform of $\frac{1}{\sqrt{2 \pi}} 1_{W}$ an s-elementary wavelet.

Received by the editors December 11, 1995 and, in revised form, May 13, 1997.

1991 Mathematics Subject Classification. Primary 46C05; Secondary 28D05, 42C15.

The author was supported in part by the NSF through the Workshop in Linear Analysis and Probability.

(C)1999 American Mathematical Society 
The importance of translations by $2 \pi$ and dilations by 2 is such that we are led to define maps $\tilde{\tau}: \mathbb{R} \rightarrow[-2 \pi,-\pi) \cup[\pi, 2 \pi)$ and $\tilde{d}: \mathbb{R}_{0}=(\mathbb{R} \backslash\{0\}) \rightarrow[-2 \pi,-\pi) \cup[\pi, 2 \pi)$ by

$$
\tilde{\tau}(x)=x+2 \pi m(x), \quad \tilde{d}(x)=2^{n(x)} x,
$$

where $m$ and $n$ are the unique integers which map $x$ into $[-2 \pi,-\pi) \cup[\pi, 2 \pi)$ under translation and dilation respectively. In this notation, $W$ is a wavelet set if and only if $\left.\tilde{d}\right|_{W}$ and $\left.\tilde{\tau}\right|_{W}$ are bijections (modulo null sets). If $\left.\tilde{d}\right|_{W}$ a nd $\left.\tilde{\tau}\right|_{W}$ are injections, modulo null sets, then we say that $W$ is a sub-wavelet set. It should be noted that not every sub-wavelet set is a subset of a wavelet set; for example, it is not hard to see that $[2 \pi, 4 \pi)$ is such a set.

We say that measurable sets $A$ and $B$ are 2-dilation congruent if there is a measurable partition $\left\{A_{n}\right\}_{n=-\infty}^{\infty}$ of $A$ such that $B=\bigcup_{n=-\infty}^{\infty} 2^{n} A_{n}$ disjointly. Similarly, $A$ and $B$ are $2 \pi$-translation congruent if there is a measurable partition $\left\{A_{n}\right\}_{n=-\infty}^{\infty}$ of $A$ such that $B=\bigcup_{n=-\infty}^{\infty} 2 \pi n+A_{n}$ disjointly.

In addition, we let $\lambda$ denote Lebesgue measure on $\mathbb{R}$ and $\mu$ denote the measure defined by $\mu(A)=\int 1_{A}(x) \frac{d \lambda}{|x|}$. Also, for any measurable subset $A$ of $\mathbb{R}$ we let $\mathcal{M}(A)$ denote the collection of all measurable subsets $B$ of $A$ such that $\lambda(B)<\infty$ and $\mu(B)<\infty$. Then,

$$
d:\left(\mathcal{M}\left(\mathbb{R}_{0}\right), \mu\right) \rightarrow(\mathcal{M}([-2 \pi,-\pi) \cup[\pi, 2 \pi)), \mu)
$$

and

$$
\tau:(\mathcal{M}(\mathbb{R}), \lambda) \rightarrow(\mathcal{M}([-2 \pi,-\pi) \cup[\pi, 2 \pi)), \lambda)
$$

defined by $A^{d}=d(A)=\tilde{d}(A)$ and $A^{\tau}=\tau(A)=\tilde{\tau}(A)$ are locally measure preserving injections (by this we mean that for any $x[$ resp. $x \neq 0]$, there is a neighborhood $U$ of $x$ such that $\left.\tau\right|_{\mathcal{M}(U), \lambda}$ [resp. $\left.\left.d\right|_{\mathcal{M}(U), \mu}\right]$ is a measure preserving injection). The maps $d$ and $\tau$ will be studied in more detail in section 2 .

Dai and Larson showed that given two wavelet sets of a certain type, it is possible to interpolate between the corresponding s-elementary wavelets. In this manner, they were able to reconstruct Meyer's wavelet. So, it is natural to ask whether it is possible to connect any two given s-elementary wavelets by a path of wavelets. In this paper we show that in fact, the collection of all s-elementary wavelets forms a path-connected subset of $L^{2}(\mathbb{R})$ by showing that the collection of all wavelet sets is path-connected in the symmetric difference metric. In particular, there is a path connecting Shannon's wavelet to Journe's wavelet, so the collection of all MRA wavelets does not form a connected component of the collection of all wavelets. This answers a question Larson posed in a seminar at Texas A\&M University in the summer of 1994.

In addition to the paper [DL], there have been two recent developments in the theory of s-elementary wavelets. Hernandez, Wang and Weiss have considered the smoothing of s-elementary wavelets in [HWW1] and [HWW2], while Fang and Wang $[\mathrm{FW}]$ have considered properties of wavelet sets as subsets of $\mathbb{R}$.

\section{Subsets of Wavelet Sets}

For motivation, we present a special case of the criterion in [DLS] for a set to be contained in a wavelet set. The construction given will be modified in section 2 to give continuity. 
Theorem 1.1. Let $A$ be a sub-wavelet set. Suppose

(1) there is an $\epsilon>0$ such that $A^{\tau} \subset[-2 \pi+\epsilon,-\pi) \cup[\pi, 2 \pi-\epsilon)$ and

(2) $S=([-2 \pi,-\pi) \cup[\pi, 2 \pi)) \backslash A^{d}$ has non-empty interior.

Then, there is a wavelet set $W \supset A$.

For the proof of Theorem 1.1 we need two lemmas.

Lemma 1.2. Let $E$ be a subset of $[-2 \pi,-\pi) \cup[\pi, 2 \pi)$ which has non-empty interior. Let $F$ be any subset of $[-2 \pi,-\pi) \cup[\pi, 2 \pi)$. Then there is a set $G$ such that the following two conditions hold.

(i) $G$ is 2-dilation congruent to a subset of $E$ and

(ii) $G$ is $2 \pi$-translation congruent to $F$.

Proof. Let $x, \epsilon$ be such that $B_{\epsilon}(x) \subset E$. Let $n$ be an integer such that $2^{n} \epsilon>6 \pi$. Then, there is an integer $m$ such that $([-2 \pi,-\pi) \cup[\pi, 2 \pi))+2 \pi m$ is contained in $2^{n} B_{\epsilon}(x)$. Let $G=F+2 \pi m$ and see that $G$ satisfies conditions (i) and (ii).

Lemma 1.3. Let $\epsilon>0$. The sets $[-2 \pi,-\pi) \cup[\pi, 2 \pi)$ and $[-\epsilon,-\epsilon / 2) \cup[\epsilon / 2, \epsilon)$ are 2dilation congruent. As a consequence, for any set $E$ a subset of $[-2 \pi,-\pi) \cup[\pi, 2 \pi)$, there is a unique $G \subset[-\epsilon,-\epsilon / 2) \cup[\epsilon / 2, \epsilon)$ such that $G$ is 2-dilation congruent to $E$.

Proof. Since $[-2 \pi,-\pi) \cup[\pi, 2 \pi)$ and $[-\epsilon,-\epsilon / 2) \cup[\epsilon / 2, \epsilon)$ are sub-wavelet sets, by Lemma 4.2 in $[\mathrm{DL}]$, it suffices to show

$$
\bigcup_{i=-\infty}^{\infty} 2^{i}([-2 \pi,-\pi) \cup[\pi, 2 \pi))=\bigcup_{i=-\infty}^{\infty} 2^{i}([-\epsilon,-\epsilon / 2) \cup[\epsilon / 2, \epsilon)) .
$$

It is easy to see that

$$
\bigcup_{i=-\infty}^{\infty} 2^{i}([-2 \pi,-\pi) \cup[\pi, 2 \pi))=\mathbb{R}=\bigcup_{i=-\infty}^{\infty} 2^{i}([-\epsilon,-\epsilon / 2) \cup[\epsilon / 2, \epsilon)) .
$$

The second statement of the lemma is now immediate.

Proof of Theorem 1.1. Write $S$ as the disjoint union of sets $\left\{E_{i}\right\}_{i=1}^{\infty}$, each of which has non-empty interior. Let $F_{1}=([-2 \pi+\epsilon,-\pi) \cup[\pi, 2 \pi-\epsilon)) \backslash A^{\tau}$. By Lemma 1.2, there is a set $G_{1}$ which is $2 \pi$-translation congruent to $F_{1}$ and 2-dilation congruent to a subset of $E_{1}$.

Use Lemma 1.3 with $E=E_{1} \backslash\left(G_{1} \cup A\right)^{d}$ to get $G_{2}$ contained in $[-\epsilon,-\epsilon / 2) \cup[\epsilon / 2, \epsilon)$ which is 2-dilation congruent to $E$.

Note that by construction, $A \cup G_{1} \cup G_{2}$ is a sub-wavelet set which satisfies condition (1) of Theorem 1.1 with $\epsilon^{\prime}=\epsilon / 2$ and condition (2) with $S=\bigcup_{i=2}^{\infty} E_{i}$.

Let $F_{2}=([-2 \pi+\epsilon / 2,-\pi) \cup[\pi, 2 \pi-\epsilon / 2)) \backslash\left(A \cup G_{1} \cup G_{2}\right)^{\tau}$. By Lemma 1.2, there is a set $G_{3}$ which is $2 \pi$-translation congruent to $F_{2}$ and 2-dilation congruent to a subset of $E_{2}$.

Use Lemma 1.3 with $E=E_{2} \backslash\left(G_{3} \cup G_{2} \cup G_{1} \cup A\right)^{d}$ to get $G_{4}$ contained in $[-\epsilon / 2,-\epsilon / 4) \cup[\epsilon / 4, \epsilon / 2)$ which is 2-dilation congruent to $E$.

Continuing in this fashion, it is easy to see that $W=A \cup\left(\bigcup_{i=1}^{\infty} G_{i}\right)$ is a wavelet set containing $A$.

Note that $A=[2 \pi, 4 \pi)$ is not a wavelet set since $A^{d}=[\pi, 2 \pi)$. (In fact, since every wavelet set must have Lebesgue measure $2 \pi, A$ is not even contained in a wavelet set.) However, by Theorem 1.1 there are wavelet sets $W_{n}$ containing 
$[2 \pi+1 / n, 4 \pi-1 / n)$ which necessarily converge to $[2 \pi, 4 \pi)$. Since it is generally easier to prove connectedness of closed sets, this partially explains why the next section is technical.

\section{Paths of Wavelet Sets}

It is shown that the wavelet sets are path-connected under the metric $d_{\lambda}(A, B)=$ $\lambda(A \triangle B)$, where $\lambda$ is Lebesgue measure on $\mathbb{R}$, which is equivalent to the $L^{2}(\mathbb{R})$ metric restricted to the s-elementary wavelets. The idea is to first connect any two wavelet sets by a path of sub-wavelet sets, then for each sub-wavelet set in the path, we will construct a containing wavelet set which depends continuously on the underlying sub-wavelet set. In order to do so, we will have to construct continuous analogues of Lemma 1.2 and Lemma 1.3.

Given a measure $m, d_{m}$ will denote the metric $d_{m}(A, B)=m(A \triangle B)$. We note here that neither the identity map

$$
I:\left(\mathcal{M}(\mathbb{R}), d_{\lambda}\right) \rightarrow\left(\mathcal{M}(\mathbb{R}), d_{\mu}\right)
$$

nor its inverse is continuous. We do, however, have the following lemma.

Lemma 2.1. Let $V$ be a measurable subset of $\mathbb{R}_{0}$ such that $\lambda(V), \mu(V)<\infty$. Then $I:\left(\mathcal{M}(V), d_{\lambda}\right) \rightarrow\left(\mathcal{M}(V), d_{\mu}\right)$ is a homeomorphism.

Proof. The statement follows from the absolute continuity of the integral, but we give a proof from the first principles to familiarize the reader with the type of argument used below.

For the continuity of $I$, it suffices to show that for every $\epsilon>0$, there is a $\delta>0$ such that whenever $\lambda(U)<\delta, \mu(U)<\epsilon$. So, fix $\epsilon>0$ and note that for all $c>0$, the set which maximizes $\{\mu(S): \lambda(S) \leq c\}$ is of the form $(-a, a) \cap V$ for some positive $a$. Now, since $\mu(V)<\infty$ and $\mu$ is non-atomic, there is some $b>0$ such that $\mu((-b, b) \cap V)<\epsilon$. Let $\delta=\lambda((-b, b) \cap V)$. Then, whenever $\lambda(U)<\delta$, we have $\mu(U) \leq \mu((-b, b) \cap V)<\epsilon$ as desired.

For the continuity of $I^{-1}$, note that for all $d>0$, the set which maximizes $\{\lambda(T)$ : $\mu(T) \leq d\}$ is of the form $((-\infty,-N] \cup[N, \infty)) \cap V$ for some non-negative $N$. Pick $N$ such that $\lambda(((-\infty,-N] \cup[N, \infty)) \cap V)<\epsilon$ and let $\delta=\mu(((-\infty,-N] \cup[N, \infty)) \cap V)$. Then, whenever $\mu(U)<\delta$, we have $\lambda(U)<\epsilon$ as desired.

An immediate consequence of Lemma 2.1 is the following lemma.

Lemma 2.2. Let $W$ be a sub-wavelet set. Then, the functions $\left.d\right|_{W}$ and $\left.\tau\right|_{W}$ : $\left(\mathcal{M}(W), d_{\lambda}\right) \rightarrow\left(\mathcal{M}([-2 \pi,-\pi) \cup[\pi, 2 \pi)), d_{\lambda}\right)$ are continuous with continuous inverses.

Proof. The function $\left.\tau\right|_{W}$ is an isometry. On the other hand, $\left.d\right|_{W}$ is an isometry from $\left(\mathcal{M}(W), d_{\mu}\right) \rightarrow\left(\mathcal{M}([-2 \pi,-\pi) \cup[\pi, 2 \pi)), d_{\mu}\right)$; hence, it is a homeomorphism. So, the lemma as stated follows from Lemma 2.1 .

The following two lemmas are continuous analogues of Lemma 1.2 and 1.3.

Lemma 2.3. Let $A \subset([-2 \pi,-\pi) \cup[\pi, 2 \pi)), s>0$, and let $N=N(A, s)$ be the unique subset of $([-s,-1 / 2 s) \cup[1 / 2 s, s))$ which is 2-dilation congruent to $A$. Then $N$ depends (jointly) continuously on $A$ and $s$ with respect to the metric $d_{\lambda}$.

Proof. The uniqueness follows from Lemma 1.3.

Write $F_{r}=\left[-r,-\frac{1}{2} r\right) \cup\left[\frac{1}{2} r, r\right)$. Then, $F_{r}^{d}=[-2 \pi,-\pi) \cup[\pi, 2 \pi)$. 
Claim. For $s, t>0$,

(1) $N\left(A \cap\left(F_{s} \cap F_{t}\right)^{d}, s\right)=N\left(A \cap\left(F_{s} \cap F_{t}\right)^{d}\right.$,t) and

(2) $N\left(A \cap\left(F_{s} \backslash F_{t}\right)^{d}, s\right) \subset F_{s} \backslash F_{t}$.

Proof of Claim. (1) $N\left(A \cap\left(F_{s} \cap F_{t}\right)^{d}, s\right)$ is the unique subset of $F_{s}$ which is 2-dilation congruent to $A \cap\left(F_{s} \cap F_{t}\right)^{d}$. Since $N\left(A \cap\left(F_{s} \cap F_{t}\right)^{d}, t\right)$ is also 2-dilation congruent to $A \cap\left(F_{s} \cap F_{t}\right)^{d}$, it suffices to show that $N\left(A \cap\left(F_{s} \cap F_{t}\right)^{d}\right.$, $\left.t\right)$ is a subset of $F_{s}$. This follows since $N\left(\left(F_{s} \cap F_{t}\right)^{d}, t\right)=F_{s} \cap F_{t} \subset F_{s}$.

(2) It suffices to show that $N\left(\left(F_{s} \backslash F_{t}\right)^{d}, s\right)=F_{s} \backslash F_{t}$. To see this, note that $F_{s} \backslash F_{t}$ is 2-dilation congruent to $\left(F_{s} \backslash F_{t}\right)^{d}$ and is a subset of $F_{s}$. Therefore, since $N\left(\left(F_{s} \backslash F_{t}\right)^{d}, s\right)$ is the unique set satisfying the conditions in the previous sentence, it follows that $N\left(\left(F_{s} \backslash F_{t}\right)^{d}, s\right)=F_{s} \backslash F_{t}$.

Returning to the proof of Lemma 2.3, write $U^{\prime}=U \cap\left(F_{s} \cap F_{t}\right)^{d}$ for $U=A, B$. Then, $A^{\prime} \cup\left(A \cap\left(F_{s} \backslash F_{t}\right)^{d}\right)=A$. So, using the definition of $N$ and property (2) above, we have

$$
\begin{aligned}
N(A, s) & =N\left(A^{\prime} \cup\left(A \cap\left(F_{s} \backslash F_{t}\right)^{d}\right), s\right) \\
& =N\left(A^{\prime}, s\right) \cup N\left(A \cap\left(F_{s} \backslash F_{t}\right)^{d}, s\right)
\end{aligned}
$$

and

$$
N(B, t)=N\left(B^{\prime}, t\right) \cup N\left(B \cap\left(F_{t} \backslash F_{s}\right)^{d}, t\right) .
$$

So, since $N\left(A \cap\left(F_{s} \backslash F_{t}\right)^{d}, s\right) \cap N\left(B \cap\left(F_{t} \backslash F_{s}\right)^{d}, t\right)=\emptyset$, we have (writing $T=$ $N(A, s) \triangle N(B, t))$

$$
\begin{aligned}
T & =\left(\left(N\left(A^{\prime}, s\right) \cup N\left(A \cap\left(F_{s} \backslash F_{t}\right)^{d}, s\right)\right) \triangle\left(N\left(B^{\prime}, t\right) \cup N\left(B \cap\left(F_{t} \backslash F_{s}\right)^{d}, t\right)\right)\right. \\
& \subset\left(N\left(A^{\prime}, s\right) \triangle N\left(B^{\prime}, t\right)\right) \cup N\left(A \cap\left(F_{s} \backslash F_{t}\right)^{d}, s\right) \cup N\left(B \cap\left(F_{t} \backslash F_{s}\right)^{d}, t\right) \\
& \subset\left(F_{s} \triangle F_{t}\right) \cup\left(N\left(A^{\prime}, s\right) \triangle N\left(B^{\prime}, t\right)\right) \\
& =\left(F_{s} \triangle F_{t}\right) \cup\left(N\left(A^{\prime}, s\right) \triangle N\left(B^{\prime}, s\right)\right) .
\end{aligned}
$$

Therefore, $N(A, s) \triangle N(B, t) \rightarrow \emptyset$ as $A \rightarrow B$ and $s \rightarrow t$ since $F_{s} \triangle F_{t} \rightarrow \emptyset$ as $s \rightarrow t$ (by definition of $F_{r}$ ) and $N\left(A^{\prime}, s\right) \triangle N\left(B^{\prime}, s\right) \rightarrow \emptyset$ as $A \rightarrow B$ by Lemma 2.2 with $W=F_{s}$. Therefore, the map $(A, s) \rightarrow N(A, s)$ is continuous.

Lemma 2.4. Let $A$ be a subset of $[-2 \pi,-\pi) \cup[\pi, 2 \pi)$, and let $\left(x_{n}\right)$ be a sequence of real numbers which decreases to $-2 \pi$ and is bounded above by $-\pi$. In addition, let $s>0$ and let $k=k(s)$ be the smallest integer such that $x_{k(s)}<-2 \pi+s$. Then, there is a function $M=M(A, s)$ such that

(i) $M$ is $2 \pi$-translation congruent to $A$

(ii) $M$ is 2-dilation congruent to a subset of $\left[x_{k(s)+1},-2 \pi+s\right)$

(iii) $M$ is a (jointly) continuous function of $A$ and $s$ with respect to the metric $d \lambda$, and

(iv) if $A_{1} \cap A_{2}=\emptyset$ and $s_{1}, s_{2}>0$, then $M\left(A_{1}, s_{1}\right)^{d} \cap M\left(A_{2}, s_{2}\right)^{d}=\emptyset$.

Proof. We give an explicit construction of $M$. For each $n$, choose the smallest integer $m_{n}$ such that $\lambda\left(2^{m_{n}}\left[x_{n+1}, x_{n}\right)\right) \geq 6 \pi$ and the smallest integer $r_{n}$ such that $([-2 \pi,-\pi) \cup[\pi, 2 \pi))+2 \pi r_{n}$ is a subset of $2^{m_{n}}\left[x_{n+1}, x_{n}\right)$. Note that $m_{n}, r_{n}$ and $x_{n}$ depend neither on $A$ nor $s$.

Let $M_{1}=M_{1}(A, s)=\left(A+2 \pi r_{k(s)-1}\right) \cap 2^{m_{k(s)-1}}\left[x_{k(s)},-2 \pi+s\right)$. Let $B=$ $B(A, s)=A \backslash M_{1}^{\tau}$, and let $M_{2}=M_{2}(A, s)=B+2 \pi r_{k(s)}$. Then, we claim that $M=M_{1} \cup M_{2}$ satisfies (i) through (iv) of Lemma 2.4. 
Conditions (i) and (ii) are immediate. Indeed, $B+2 \pi r_{k(s)} \subset([-2 \pi,-\pi) \cup$ $[\pi, 2 \pi))+2 \pi r_{k(s)} \subset 2^{m_{k(s)}}\left[x_{k(s)+1}, x_{k(s)}\right)$. Therefore, (ii) follows from the fact $\left[x_{k(s)+1}, x_{k(s)}\right) \cap\left[x_{k(s)},-2 \pi+s\right)=\emptyset$.

To prove (iii), let $A_{n} \rightarrow A$ and $t_{n} \rightarrow t$. There are two cases to consider. First, suppose that $k\left(t_{n}\right) \rightarrow k(t)$ (since $k$ is integer valued, this says $k\left(t_{n}\right)=k(t)$ for large $n)$. It suffices to show that $M_{1}\left(A_{n}, t_{n}\right) \rightarrow M_{1}(A, t)$ and $M_{2}\left(A_{n}, t_{n}\right) \rightarrow M_{2}(A, t)$. To see this, we have

$$
\begin{aligned}
M_{1}\left(A_{n}, t_{n}\right) & =\left(A_{n}+2 \pi r_{k\left(t_{n}\right)-1}\right) \cap 2^{m_{k\left(t_{n}\right)-1}\left[x_{k\left(t_{n}\right)},-2 \pi+t_{n}\right)} \\
& \rightarrow\left(A+2 \pi r_{k(t)-1}\right) \cap 2^{m_{k(t)-1}}\left[x_{k(t)},-2 \pi+t\right) \\
& =M_{1}(A, t) .
\end{aligned}
$$

So, $B\left(A_{n}, t_{n}\right)=A_{n} \backslash M_{1}\left(A_{n}, t_{n}\right)^{\tau} \rightarrow A \backslash M_{1}(A, t)^{\tau}=B(A, t)$. Thus, $M_{2}\left(A_{n}, t_{n}\right)=$ $B\left(A_{n}, t_{n}\right)+2 \pi r_{k\left(t_{n}\right)} \rightarrow B(A, t)+2 \pi r_{k(t)}=M_{2}(A, t)$, as desired.

Now suppose that $k\left(t_{n}\right) \nrightarrow k(t)$. By definition of $k$, this can only occur when $-2 \pi+t=x_{m}$ for some $m$. In addition, if $t_{n}$ increases to $t$, then $k\left(t_{n}\right) \rightarrow k(t)$, so we can assume that $t_{n}$ decreases to $t$. Note that then $k\left(t_{n}\right) \rightarrow k(t)-1$, so we may assume that $k\left(t_{n}\right)=k(t)-1$. Then,

$$
\begin{aligned}
M_{1}\left(A_{n}, t_{n}\right) & =\left(A_{n}+2 \pi r_{k\left(t_{n}\right)-1}\right) \cap 2^{m_{k\left(t_{n}\right)-1}}\left[x_{k\left(t_{n}\right)},-2 \pi+t_{n}\right) \\
& =\left(A_{n}+2 \pi r_{k(t)-2}\right) \cap 2^{m_{k(t)-2}}\left[x_{k(t)-1},-2 \pi+t_{n}\right) \\
& \rightarrow\left(A+2 \pi r_{k(t)-2}\right) \cap 2^{m_{k(t)-2}}\left[x_{k(t)-1},-2 \pi+t\right)=\emptyset .
\end{aligned}
$$

(Since $k(t)$ is the smallest natural number such that $x_{k(t)}<-2 \pi+t$, we have $x_{k(t)-1} \geq-2 \pi+t$. Therefore, $\left[x_{k(t)-1},-2 \pi+t\right)=\emptyset$ modulo a null set.) So, $M\left(A_{n}, t_{n}\right)=M_{2}\left(A_{n}, t_{n}\right)=\left(A_{n} \backslash M_{1}^{\tau}\right)+2 \pi r_{k\left(t_{n}\right)} \rightarrow A+2 \pi r_{k(t)-1}$. On the other hand,

$$
\begin{aligned}
M_{1}(A, t) & =\left(A+2 \pi r_{k(t)-1}\right) \cap 2^{m_{k(t)-1}\left[x_{k(t)},-2 \pi+t\right)} \\
& =A+2 \pi r_{k(t)-1} .
\end{aligned}
$$

Indeed, since $-2 \pi+t=x_{m}$ for some $m,([-2 \pi,-\pi) \cup[\pi, 2 \pi))+2 \pi r_{k(t)-1} \subset$ $\left[x_{k(t)},-2 \pi+t\right)$. Therefore, $M(A, t)=M_{1}(A, t)=A+2 \pi r_{k(t)-1}$. So, we have $M\left(A_{n}, t_{n}\right) \rightarrow M(A, t)$ and (iii) is proved.

To prove (iv), note that by construction we can write $M\left(A_{1}, s_{1}\right)=\bigcup_{n=0}^{\infty} 2^{m_{n}} G_{n}$ and $M\left(A_{2}, s_{2}\right)=\bigcup_{n=0}^{\infty} 2^{m_{n}} H_{n}$ with $G_{n}, H_{n} \subset\left[x_{n+1}, x_{n}\right)$ and $x_{0}=-\pi$. Then, $M\left(A_{1}, s_{1}\right)^{d}=\bigcup_{n=0}^{\infty} G_{n}$ and $M\left(A_{2}, s_{2}\right)^{d}=\bigcup_{n=0}^{\infty} H_{n}$, so $M\left(A_{1}, s_{1}\right)^{d} \cap M\left(A_{2}, s_{2}\right)^{d}=$ $\bigcup_{n=0}^{\infty}\left(H_{n} \cap G_{n}\right)$. Therefore, it suffices to show that $G_{n} \cap H_{n}=\emptyset$ for all $n$. But, this follows from the fact that $2^{m_{n}} G_{n}-2 \pi r_{n} \subset A_{1}, 2^{m_{n}} H_{n}-2 \pi r_{n} \subset A_{2}$ and $A_{1} \cap A_{2}=\emptyset$.

We are now ready for the main result of this paper.

Theorem 2.5. The wavelet sets are path-connected in the symmetric difference metric.

Proof. It is shown that every wavelet set $W$ is path-connected to $[-2 \pi,-\pi) \cup[\pi, 2 \pi)$. The first step is to construct a path of subsets of wavelet sets, then for each set in the path, we will find a wavelet superset which depends continuously on the subset. So, for $0 \leq t \leq \pi$ let $R_{t}$ be the subset of $W$ which is $2 \pi$-translation congruent to $[-\pi-t,-\pi) \cup[\pi, \pi+t)$, i.e. $R_{t}^{\tau}=[-\pi-t,-\pi) \cup[\pi, \pi+t)$. Let $P_{t}$ 
be the subset of $W$ 2-dilation congruent to $[-2 \pi,-2 \pi+t) \cup[2 \pi-t, 2 \pi)$ and let $Q_{t}=[-2 \pi+t,-\pi-t) \cup[\pi+t, 2 \pi-t)$. Then the path of sets defined by

$$
S_{t}= \begin{cases}{\left[\left(Q_{t} \cup R_{t}\right) \backslash\left(R_{t}^{d} \cap Q_{t}\right)\right] \backslash P_{t}} & \text { if } 0 \leq t \leq \pi / 2, \\ R_{t} \backslash P_{\pi-t}, & \text { if } \pi / 2 \leq t \leq \pi\end{cases}
$$

is a path of subsets of wavelet sets which connect $E$ to $W$ and which are of the type mentioned in Proposition 1.1 for $0<t<\pi$. Note that the path is continuous by the continuity of dilations, translations and set operations with respect to $d_{m}$.

The second step is a recursive construction of sets $M_{i}$, continuous functions of $t$, such that $S_{t} \cup\left(\bigcup_{i=0}^{\infty} M_{i}\right)$ is a wavelet set for each $t$. Fix $x_{n}$ and the function $k$ as in Lemma 2.4. Also, define $C_{i}=C_{i}(t)$ to be $\left[-2 \pi+t / 2^{i},-2 \pi+t / 2^{i-1}\right) \cup$ $\left[2 \pi-t / 2^{i}, 2 \pi-t / 2^{i-1}\right)$ for $i=1,2,3, \ldots$ and $C_{0}$ to be $[-2 \pi+t,-\pi) \cup[\pi, 2 \pi-t)$. For notational convenience in what follows, we denote

$$
t^{\prime}= \begin{cases}t & \text { if } 0 \leq t \leq \pi / 2 \\ \pi-t & \text { if } \pi / 2 \leq t \leq \pi\end{cases}
$$

Finally, recall the definitions of the functions $N$ and $M$ from Lemma 2.3 and 2.4 respectively.

Notice that we have the following relations.

(1) $C_{0}\left(t^{\prime}\right) \supset S_{t}^{d}$,

(2) $C_{0}\left(t^{\prime}\right) \supset S_{t}^{\tau}$.

Now, let $A_{0}=A_{0}(t)=C_{0}\left(t^{\prime}\right) \backslash S_{t}^{d}, s_{0}=s_{0}(t)=t^{\prime}$ and define $M_{0}=N\left(A_{0}, s_{0}\right)$, which is a continuous function of $t$ by Lemma 2.3. Note that

(1) $\left[x_{k\left(t^{\prime}\right)+1},-\pi\right) \cup\left[\pi,-x_{k\left(t^{\prime}\right)}\right) \supset\left(M_{0} \cup S_{t}\right)^{d} \supset C_{0}\left(t^{\prime}\right)$, and

(2) $C_{0}\left(t^{\prime}\right) \cup C_{1}\left(t^{\prime}\right) \supset\left(M_{0} \cup S_{t}\right)^{\tau}$.

Then let $B_{0}=B_{0}(t)=C_{0}\left(t^{\prime}\right) \backslash\left(M_{0} \cup S_{t}\right)^{\tau}, r_{0}=t^{\prime}$ and define $M_{1}=M\left(B_{0}, r_{0}\right)$, which is a continuous function of $t$ by Lemma 2.4. Note that

(1) $\left[x_{k\left(t^{\prime}\right)+1},-\pi\right) \cup\left[\pi,-x_{k\left(t^{\prime}\right)+1}\right) \supset\left(M_{0} \cup M_{1} \cup S_{t}\right)^{d} \supset C_{0}\left(t^{\prime}\right)$, and

(2) $C_{0}\left(t^{\prime}\right) \cup C_{1}\left(t^{\prime}\right) \supset\left(M_{0} \cup M_{1} \cup S_{t}\right)^{\tau} \supset C_{0}\left(t^{\prime}\right)$.

For the recursive definition, suppose we have $M_{0}, \ldots, M_{2 p-1}$ defined such that each is a continuous function of $t$ and for all $r$ between 0 and $p-1$ the following conditions hold.

(1a) $\left[x_{k\left(t^{\prime} / 2^{r}\right)+1},-\pi\right) \cup\left[\pi,-x_{k\left(t^{\prime} / 2^{r}\right)+1}\right) \supset\left(\bigcup_{i=0}^{2 r} M_{i} \cup S_{t}\right)^{d} \supset \bigcup_{i=0}^{r} C_{i}\left(t^{\prime}\right)$, and

(1b) $\bigcup_{i=0}^{r+1} C_{i} a\left(t^{\prime}\right) \supset\left(\bigcup_{i=0}^{2 r} M_{i} \cup S_{t}\right)^{\tau} \supset \bigcup_{i=0}^{r-1} C_{i}\left(t^{\prime}\right)$;

(2a) $\left[x_{k\left(t^{\prime} / 2^{r}\right)+1},-\pi\right) \cup\left[\pi,-x_{k\left(t^{\prime} / 2^{r}\right)+1}\right) \supset\left(\bigcup_{i=0}^{2 r+1} M_{i} \cup S_{t}\right)^{d} \supset \bigcup_{i=0}^{r} C_{i}\left(t^{\prime}\right)$, and

(2b) $\bigcup_{i=0}^{r+1} C_{i}\left(t^{\prime}\right) \supset\left(\bigcup_{i=0}^{2 r+1} M_{i} \bigcup S_{t}\right)^{\tau} \supset \bigcup_{i=0}^{r} C_{i}\left(t^{\prime}\right)$.

Then, we need to define $M_{2 p}, M_{2 p+1}$ having the same properties. Let $A_{p}=$ $C_{p}\left(t^{\prime}\right) \backslash\left(\bigcup_{i=1}^{2 p-1} M_{i} \cup S_{t}\right)^{d}, s_{p}=t^{\prime} / 2^{p}$ and define $M_{2 p}=N\left(A_{p}, s_{p}\right)$, which is a continuous function of $t$. Let $B_{p}=C_{p}\left(t^{\prime}\right) \backslash\left(\bigcup_{i=1}^{2 p} M_{i} \cup S_{t}\right)^{\tau}, r_{p}=t^{\prime} / 2^{p}$, and define $M_{2 p+1}=M\left(B_{p}, r_{p}\right)$, which is a continuous function of $t$. Note that $M_{2 p+1}^{d}$ has empty intersection with $M_{i}^{d}$ for $i \leq 2 p$ by Lemma 2.4 when $i$ is odd and by construction when $i$ is even. Thus, we have defined wavelet sets $W_{t}=\bigcup_{i=0}^{\infty} M_{i} \cup S_{t}$.

In order to see that this is a continuous construction, note that

$$
m\left(\bigcup_{i=2 r+1}^{\infty} M_{i}\right) \leq t^{\prime} / 2^{r}
$$


by condition (2) and the definition of $C_{i}$. This estimate together with the continuity of the pieces gives the continuity of $W_{t}$.

Corollary 2.6. The s-elementary wavelets form a path-connected subset of $L^{2}(\mathbb{R})$.

Proof. This follows immediately from Theorem 2.5 and the fact that $d_{\lambda}$ is equivalent to the $L^{2}(\mathbb{R})$ metric restricted to the s-elementary wavelets.

\section{Generalizations to $\mathbb{R}^{n}$}

We begin with the definition of an $n$-dimensional wavelet set. Let $D: \mathbb{R}^{n} \rightarrow \mathbb{R}^{n}$ be a bijective linear transform whose inverse has norm less than 1 , and let $\left\{T_{i}: i=\right.$ $-\infty \ldots \infty\}$ be the translations by multiples of $2 \pi$ in every coordinate direction, i.e. $T_{i}\left(x_{1}, \ldots, x_{n}\right)=\left(x_{1}+2 \pi k_{1, i}, \ldots, x_{n}+2 \pi k_{n, i}\right)$. Then, we say a measurable set $W$ is an $n$-dimensional wavelet set if $(2 \pi)^{-n / 2} 1_{W}$ is the Fourier transform of a wavelet $f$, i.e. $\left\{(\operatorname{det}(D))^{m / 2} f\left(D^{m} x+\left(k_{1}, \ldots, k_{n}\right): m, k_{1}, \ldots, k_{n} \in \mathbb{Z}\right\}\right.$ is an orthonormal basis for $\mathbb{R}^{n}$. We call this inverse Fourier transform an $n$-dimensional s-elementary wavelet, where $D$ is understood.

As was shown in [DLS], a set $W$ is an $n$-dimensional wavelet set if and only if

$$
\bigcup_{i=-\infty}^{\infty} D^{i}(W)=\mathbb{R}^{n} \text { and } \bigcup_{i=-\infty}^{\infty} T_{i}(W)=\mathbb{R}^{n},
$$

with both unions disjoint. The proof of the existence of such sets is given in [DLS], and the idea is similar to the proof of Theorem 1.1.

The proof that the $n$-dimensional wavelet sets are path-connected is largely a matter of developing the correct analogues to the 1-dimensional case. Therefore, we will omit some details when convenient. (The full proof appears in the author's dissertation [S].) For sets $A, B \subset \mathbb{R}^{n}$ with $\operatorname{conv}(A) \subset \operatorname{conv}(B)$, we define the annulus $\mathcal{A}(A, B)$ to be $\operatorname{conv}(B) \backslash \operatorname{conv}(A)$. As suggested by the name, we will be mostly concerned with the case that $A$ and $B$ are ellipsoids.

Let $S^{n}$ denote the unit sphere in $\mathbb{R}^{n}$ and consider $\mathcal{A}\left(S^{n}, D\left(S^{n}\right)\right.$ ). (Note that since $\left\|D^{-1}\right\|<1, \mathcal{A}\left(S^{n}, D\left(S^{n}\right)\right)$ is not empty.) Now, let $\left(w_{1}, \ldots, w_{n}\right)$ be orthonormal axes of $D\left(S^{n}\right)$; in other words, there exist constants $\left(a_{1}, \ldots, a_{n}\right)$ such that $a_{i}>1$ and $D\left(S^{n}\right)=\left\{\left(x_{1}, \ldots, x_{n}\right): \sum_{i=1}^{n} \frac{x_{i}^{2}}{a_{i}^{2}}=1\right\}$, where $\left(x_{1}, \ldots, x_{n}\right)$ is the coordinatization of a point in $\mathbb{R}^{n}$ with respect to the orthonormal basis $\left(w_{1}, \ldots, w_{n}\right)$. Define $f_{i}:[1,2] \rightarrow\left[1, a_{i}\right]$ by $f_{i}(s)=(2-s)+(1-s) a_{i}$, and for $1 \leq s \leq 2$, let $P_{s}$ be the ellipsoid defined by $P_{s}=\left\{\left(x_{1} \ldots, x_{n}\right): \sum_{i=1}^{n} \frac{x_{i}^{2}}{f_{i}(s)^{2}}=1\right\}$.

We extend $P_{s}$ to the positive axis as follows. Let $s>0$ and let $n$ be the unique integer such that $2^{n} t \in(1,2]$. Define $P_{s}=D^{-n}\left(P_{2^{n}}\right)$. Finally, we define $P_{0}=\{0\}$.

As in the one-dimensional case, we say that $E, F \subset \mathbb{R}^{n}$ are $D$-congruent if there is a partition $\left\{E_{i}\right\}_{i=-\infty}^{\infty}$ of $E$ such that

$$
\bigcup_{i=-\infty}^{\infty} D^{i}\left(E_{i}\right)=F
$$

Similarly, we say $E, F \subset \mathbb{R}^{n}$ are $T$-congruent if there is a partition $\left\{E_{i}\right\}_{i=-\infty}^{\infty}$ of $E$ such that

$$
\bigcup_{i=-\infty}^{\infty} T_{i}\left(E_{i}\right)=F
$$


Also, given wavelet sets $W$ and $E \subset W$, we define $E^{\tau}$ to be the subset of $[-\pi, \pi]^{n}$ which is $T$-congruent to $E$ and $E^{d}$ to be the subset of $\mathcal{A}\left(S^{n}, D\left(S^{n}\right)\right)$ which is $D$-congruent to $E$. One can check that since $D$ and $D^{-1}$ are bounded, and all wavelet sets have finite Lebesgue measure, the functions $E \rightarrow E^{d}$ and $E \rightarrow E^{\tau}$ are continuous with continuous inverses when restricted to subsets of a given wavelet set.

We now give the $n$-dimensional analogues to Lemmas 2.3 and 2.4.

Lemma 3.1. Let $A \subset \mathcal{A}\left(P_{\pi}, P_{2 \pi}\right), s>0$. Then there is a unique subset $N(A, s)$ of $\mathcal{A}\left(P_{s / 2}, P_{s}\right)$ which is $D$ congruent to $A$. Furthermore, $N$ is a jointly continuous function of $A$ and $s$.

Proof. The uniqueness follows from the fact that $\mathcal{A}\left(P_{s / 2}, P_{s}\right)$ is a $D$-dilation generator of $\mathbb{R}^{n}$. (By this, we mean that $\bigcup_{i=-\infty}^{\infty} D^{i}\left(\mathcal{A}\left(P_{s / 2}, P_{s}\right)\right)=\mathbb{R}^{n}$, with the union disjoint.) We will, however, need the explicit formula for $N$, so we give it here. Let $n=n(s)$ be the unique integer such that $\pi \leq 2^{n} s<2 \pi$. Then,

$$
N(A, s)=D^{-(n+1)}\left(\mathcal{A}\left(P_{2^{n} s}, P_{2 \pi}\right) \cap A\right) \cup D^{-n}\left(\mathcal{A}\left(P_{\pi}, P_{2^{n} s}\right) \cap A\right) .
$$

To prove continuity, let $A_{m} \rightarrow A$ and $s_{m} \rightarrow s$. Then, there are two cases to consider. First, suppose $n\left(s_{m}\right) \rightarrow n(s)$. Then, we may assume that $n\left(s_{m}\right)=n(s)$ $=n$. So, the continuity follows from the continuity of unions, intersections, $P_{s}$ and D.

The second case is that $n\left(s_{m}\right) \nrightarrow n(s)$. By passing to a subsequence if necessary, we may assume $\pi \leq 2^{n\left(s_{m}\right)} s<2 \pi$ and $2^{n\left(s_{m}\right)} s \rightarrow 2 \pi$. Then, for all $m, 2^{n\left(s_{m}\right)} s=2 \pi$, $n(s)=n\left(s_{m}\right)-1$ and $2^{n(s)}=\pi$. We write $n=n(s)$. So,

$$
\begin{aligned}
N\left(A_{m}, s_{m}\right) & =D^{-n-2}\left(\mathcal{A}\left(P_{2^{n+1} s_{m}}, P_{2 \pi}\right) \cap A_{m}\right) \cup D^{-n-1}\left(\mathcal{A}\left(P_{\pi}, P_{2^{n} s_{m}}\right) \cap A_{m}\right) \\
& \rightarrow \emptyset \cup D^{-(n+1)}\left(\mathcal{A}\left(P_{\pi}, P_{2 \pi}\right) \cap A\right) \\
& =N(A, s),
\end{aligned}
$$

as desired.

Lemma 3.2. Let $A$ be a subset of $[-\pi, \pi)^{n}$ and let $\left\{x_{m}\right\}_{m=1}^{\infty}$ be a sequence of real numbers which decreases to $\pi$ and is bounded above by $2 \pi$. In addition, let $\pi>s>0$, and let $k=k(s)$ be the smallest integer such that $x_{k(s)}<\pi+s$. Then, there is a function $M=M(A, s)$ such that

(i) $M$ is $T$-congruent to $A$,

(ii) $M$ is D-congruent to a subset of $\mathcal{A}\left(P_{x_{k(s)+1}}, P_{\pi+s}\right)$,

(iii) $M$ is a jointly continuous function of $A$ and $s$, and

(iv) if $A_{1} \cap A_{2}=\emptyset$ and $s_{1}, s_{2}>0$, then $M\left(A_{1}, s_{1}\right)^{d} \cap M\left(A_{2}, s_{2}\right)^{d}=\emptyset$.

Proof. We define $M$ explicitly as in the proof of Lemma 2.4. For each natural number $j$, let $m_{j}$ be a large enough integer such that there is an $r_{j}$ with

$$
T_{r_{j}}\left([-\pi, \pi)^{n}\right) \subset D^{m_{j}}\left(\mathcal{A}\left(P_{x_{j+1}}, P_{x_{j}}\right)\right) .
$$

To define $M$, let $M_{1}=M_{1}(A, s)=T_{r_{k(s)-1}}(A) \cap D^{m_{k(s)-1}}\left(\mathcal{A}\left(P_{x_{k(s)}}, P_{\pi+s}\right)\right)$. Let $E$ be the subset of $A$ which is $T$-congruent to $M_{1}(A, s)$. Define $B=B(A, s)=A \backslash E$. Let $M_{2}=M_{2}(A, s)=T_{r_{k(s)}}(B)$. Then $M=M_{1} \cup M_{2}$ satisfies conditions (i) through (iv) of Lemma 3.2.

The proof that $M$ satisfies the above conditions is identical to the proof of Lemma 2.4 with $T$ playing the role of translation, $D$ playing the role of dilation, and $P_{x_{n}}$ playing the role of $x_{n}$, so we omit the details. 
Theorem 3.3. The collection of all n-dimensional wavelet sets is path-connected in the symmetric difference metric.

Proof. Let $W_{1}, W_{2}$ be wavelet sets. The first step is to construct a path of subwavelet sets which connects $W_{1}$ to $W_{2}$. Let $0<t_{0} \leq \pi / 2$ be small enough so that $P_{t_{0}} \subset[-\pi, \pi]^{n}$. (This is possible since $\left\|D^{-1}\right\|<1$.) Let $B_{t}=[-t, t]^{n}$. For $0 \leq t \leq \pi$, we have the following definitions.

$O_{t}$ is the subset of $W_{1}$ such that $O_{t}^{\tau}=B_{\pi} \backslash B_{t}$,

$Q_{t}$ is the subset of $W_{2}$ such that $Q_{t}^{\tau}=B_{t}$,

$R_{t}$ is the subset of $W_{1}$ such that $R_{t}^{d}=\mathcal{A}\left(P_{\pi}, P_{\pi+\min \left(t, t_{0}\right)}\right)$,

$X_{t}$ is the subset of $W_{2}$ such that $X_{t}^{d}=\mathcal{A}\left(P_{\pi}, P_{\pi+\min \left(t, t_{0}\right)}\right)$,

$U_{t}$ is the subset of $W_{1}$ such that $U_{t}^{d}=O_{t}^{d} \cap Q_{t}^{d}$, and

$V_{t}$ is the subset of $O_{t} \cup Q_{t}$ such that $V_{t}^{\tau}=\mathcal{A}\left(\{0\}, P_{\min \left(t, t_{0}\right)}\right)$.

Then, we have that $S_{t}$ defined by

$$
S_{t}= \begin{cases}\left(O_{t} \cup Q_{t}\right) \backslash\left(R_{t} \cup X_{t} \cup U_{t} \cup V_{t}\right) & \text { if } 0 \leq t \leq \pi / 2, \\ \left(O_{t} \cup Q_{t}\right) \backslash\left(R_{\pi-t} \cup X_{\pi-t} \cup U_{t} \cup V_{\pi-t}\right) & \text { if } \pi / 2 \leq t \leq \pi\end{cases}
$$

is a path of sub-wavelet sets connecting $W_{1}$ to $W_{2}$ by the continuity of $E \rightarrow E^{d}$ and $E \rightarrow E^{\tau}$, Lemma 3.1 and Lemma 3.2.

Now, we will construct sets $\left\{M_{i}\right\}_{i=0}^{\infty}$ which are continuous functions of $t$ such that $S_{t} \cup_{i=0}^{\infty} M_{i}(t)$ is a wavelet set for each $t$.

Fix $\left\{x_{n}\right\}_{n=1}^{\infty}$ and the function $k$ as in Lemma 3.2. Define $C_{i}=C_{i}(t)$ to be $\mathcal{A}\left(P_{\pi+t / 2^{i}}, P_{\pi+t / 2^{i-1}}\right)$ for $i$ a natural number and $C_{0}$ to be $\mathcal{A}\left(P_{\pi+t}, P_{2 \pi}\right)$. Define $t^{\prime}$ by

$$
t^{\prime}= \begin{cases}t & \text { if } t \leq t_{0} \\ t_{0} & \text { if } t_{0} \leq t \leq \pi-t_{0} \\ \pi-t & \text { if } \pi-t_{0} \leq t \leq \pi\end{cases}
$$

Finally, recall the definitions of $N$ and $M$ in Lemma 3.1 and Lemma 3.2.

Now, let $A_{0}=A_{0}(t)=C_{0}\left(t^{\prime}\right) \backslash S_{t}^{d}, s_{0}=s_{0}(t)=t^{\prime}$ and define $M_{0}=M_{0}(t)=$ $N\left(A_{0}, s_{0}\right)$, which is a continuous function of $t$ by Lemma 3.1. Note

(1) $\mathcal{A}\left(P_{\pi+t^{\prime}}, P_{2 \pi}\right) \supset\left(M_{0} \cup S_{t}\right)^{d}=C_{0}\left(t^{\prime}\right)$, and

(2) $[-\pi, \pi]^{n} \backslash \mathcal{A}\left(0, P_{t^{\prime} / 2}\right) \supset\left(M_{0} \cup S_{t}\right)^{\tau}$.

Then let $B_{0}=B_{0}(t)=\left([-\pi, \pi]^{n} \backslash \mathcal{A}\left(0, P_{t^{\prime} / 2}\right)\right) \backslash\left(M_{0} \cup S_{t}\right)^{\tau}, r_{0}=t^{\prime}$ and define $M_{1}=M\left(B_{0}, r_{0}\right)$, which is a continuous function of $t$ by Lemma 3.2. Note that

(1) $\mathcal{A}\left(P_{k\left(t^{\prime}\right)+1}, P_{2 \pi}\right) \supset\left(M_{0} \cup M_{1} \cup S_{t}\right)^{d} \supset C_{0}\left(t^{\prime}\right)$, and

(2) $\left(M_{0} \cup M_{1} \cup S_{t}\right)^{\tau}=[-\pi, \pi]^{n} \backslash \mathcal{A}\left(0, P_{t^{\prime} / 2}\right)$.

Now, let $A_{1}=C_{1}\left(t^{\prime}\right) \backslash\left(M_{0} \cup M_{1} \cup S_{t}\right)^{d}$ and $s_{1}=t^{\prime} / 2$. Define $M_{2}=N\left(A_{1}, s_{1}\right)$. Then,

(1) $\mathcal{A}\left(P_{k\left(t^{\prime} / 2\right)+1}, P_{2 \pi}\right) \supset\left(\bigcup_{i=0}^{2} M_{i} \cup S_{t}\right)^{d} \supset C_{0}\left(t^{\prime}\right) \cup C_{1}\left(t^{\prime}\right)$, and

(2) $[-\pi, \pi]^{n} \backslash \mathcal{A}\left(0, P_{t^{\prime} / 4}\right) \supset\left(\bigcup_{i=0}^{2} M_{i} \cup S_{t}\right)^{\tau} \supset[-\pi, \pi]^{n} \backslash \mathcal{A}\left(0, P_{t^{\prime} / 2}\right)$.

Let $B_{1}=\left([-\pi, \pi]^{n} \backslash \mathcal{A}\left(0, P_{t^{\prime} / 4}\right)\right) \backslash\left(\bigcup_{i=0}^{2} M_{i} \cup S_{t}\right)^{\tau}$ and $r_{1}=t^{\prime} / 2$. Define $M_{3}=$ $M\left(B_{1}, r_{1}\right)$. Note that

(1) $\mathcal{A}\left(P_{k\left(t^{\prime} / 2\right)+1}, P_{2 \pi}\right) \supset\left(\bigcup_{i=0}^{3} M_{i} \cup S_{t}\right)^{d} \supset C_{0}\left(t^{\prime}\right) \cup C_{1}\left(t^{\prime}\right)$, and

(2) $\left(M_{0} \cup M_{1} \cup S_{t}\right)^{\tau}=[-\pi, \pi]^{n} \backslash \mathcal{A}\left(0, P_{t^{\prime} / 4}\right)$. 
Note that $M_{3}^{d} \cap M_{i}^{d}=\emptyset$ for $i<3$ by construction when $i$ is even, and by Lemma 3.2 (v) when $i$ is odd.

Continuing in this fashion, one can check as in the one-dimensional case that the resulting sets $L_{t}=\bigcup_{i=0}^{\infty} M_{i} \cup S_{t}$ forms a path of wavelet sets connecting $W_{1}$ to $W_{2}$.

\section{ACKNOWLEDGMENTS}

The author would like to thank W. B. Johnson and D. R. Larson for their many helpful comments, and G. Garrigos for his careful proofreading.

\section{REFERENCES}

[C] C. K. Chui, An Introduction to Wavelets, Acad. Press, New York, 1992. MR 93f:42055

[DL] X. Dai and D. Larson, Wandering vectors for unitary systems and orthogonal wavelets, Mem. Amer. Math. Soc., to appear. CMP 97:07

[DLS] X. Dai, D. Larson and D. Speegle, Wavelets in $\mathbb{R}^{n}$, J. Fourier Anal. Appl. 3 (1997), 451-456. CMP 97:17

[FW] X. Fang and X. Wang, Construction of minimally supported frequency wavelets, J. Fourier Anal. Appl. 2 (1996), no. 4, 315-327. MR 97d:42030

[H] P. Halmos, A Hilbert Space Problem Book, second ed., Springer-Verlag, New York, 1982. MR 84e: 47001

[HWW1] E. Hernandez, X. Wang and G. Weiss, Smoothing minimally supported wavelets. I, J. Fourier Anal. Appl. 2 (1996), no. 2, 329-340. MR 97h:42015

[HWW2] E. Hernandez, X. Wang and G. Weiss, Smoothing minimally supported wavelets. II, J. Fourier Anal. Appl. 2 (1997), no. 1, 23-41. CMP 97:06

[S] Darrin Speegle, $S$-elementary wavelets and the into $C(K)$ extension property, Dissertation, Texas A\&M University.

Department of Mathematics, Texas A\&M University, College Station, Texas 77843

E-mail address: speegle@math.tamu.edu

Current address: Department of Mathematics, Saint Louis University, St. Louis, Missouri 63103 